\title{
Novel Lithium-Ion Capacitor Based on a NiO-rGO Composite
}

\author{
Qi An ${ }^{1, *}$, Xingru Zhao ${ }^{2}$, Shuangfu Suo ${ }^{1}$ and Yuzhu Bai ${ }^{1}$ \\ 1 Department of Mechanical Engineering, Tsinghua University, Beijing 100084, China; \\ sfsuo@tsinghua.edu.cn (S.S.); baiyuzhu403@163.com (Y.B.) \\ 2 Beijing Institute of Nanoenergy and Nanosystems, Chinese Academy of Sciences, Beijing 101400, China; \\ zhaoxingru@binn.cas.cn \\ * Correspondence: thaq@mail.tsinghua.edu.cn
}

\section{check for}

updates

Citation: An, Q.; Zhao, X.; Suo, S.; Bai, Y. Novel Lithium-Ion Capacitor Based on a NiO-rGO Composite. Materials 2021, 14, 3586. https:/ / doi.org/10.3390/ma14133586

Academic Editor: Suzy Surblé

Received: 23 May 2021

Accepted: 23 June 2021

Published: 27 June 2021

Publisher's Note: MDPI stays neutral with regard to jurisdictional claims in published maps and institutional affiliations.

\begin{abstract}
Lithium-ion capacitors (LICs) have been widely explored for energy storage. Nevertheless, achieving good energy density, satisfactory power density, and stable cycle life is still challenging. For this study, we fabricated a novel LIC with a NiO-rGO composite as a negative material and commercial activated carbon (AC) as a positive material for energy storage. The NiO-rGO// AC system utilizes $\mathrm{NiO}$ nanoparticles uniformly distributed in $\mathrm{rGO}$ to achieve a high specific capacity (with a current density of $0.5 \mathrm{~A} \mathrm{~g}^{-1}$ and a charge capacity of $945.8 \mathrm{~mA} \mathrm{~h} \mathrm{~g}^{-1}$ ) and uses AC to provide a large specific surface area and adjustable pore structure, thereby achieving excellent electrochemical performance. In detail, the NiO-rGO/ / AC system (with a mass ratio of 1:3) can achieve a high energy density $\left(98.15 \mathrm{~W} \mathrm{~h} \mathrm{~kg}^{-1}\right)$, a high power density $\left(10.94 \mathrm{~kW} \mathrm{~kg}^{-1}\right)$, and a long cycle life (with $72.1 \%$ capacity retention after 10,000 cycles). This study outlines a new option for the manufacture of LIC devices that feature both high energy and high power densities.
\end{abstract}

Keywords: lithium-ion capacitors; NiO-rGO composite; energy density; power density

\section{Introduction}

With the ongoing energy crisis and international impetus to mitigate environmental pollution and climate change, energy storage devices that serve as intermediaries of clean and efficient energy are attracting ever more attention among researchers [1-7]. Energy storage devices such as lithium-ion batteries (LIBs) and supercapacitors (SCs) are widely used due to their satisfactory electrochemical performance [7-9]. The competitive advantage of LIBs is their high energy density (i.e., $150-200 \mathrm{~W} \mathrm{~h} \mathrm{~kg}^{-1}$ ). However, their power density is fairly low (less than $100 \mathrm{~W} \mathrm{~h} \mathrm{~kg}^{-1}$ ) and a poor cycle life limits their practical applications. Meanwhile, SCs can offer high power density $\left(10 \mathrm{~kW} \mathrm{~kg}^{-1}\right)$ and excellent cycle stability, but feature low energy density (5-10 $\mathrm{W} \mathrm{h} \mathrm{kg} \mathrm{kg}^{-1}$ ), impeding their utility as singular energy storage devices [10-12]. These differences derive from the different energy storage mechanisms of these systems; LIBs store energy by inserting lithium ions into, or extracting them from, most electrodes, whereas SCs store energy through the adsorption/desorption of ions on the electrode surface [13].

To maximize their electrochemical performance, lithium-ion capacitors (LICs) have been widely studied. The main factor affecting the performance of LICs is the mismatch of reaction kinetics between the two electrodes; the parameter with the greatest influence in this regard is the electrode material [14-18]. Therefore, the identification of electrode materials with a suitable specific capacity, remarkable rate performance, and excellent stability has become a key challenge. With respect to the positive electrode, carbon-derived materials (activated carbon (AC) [18], graphene [19], and metal-organic framework (MOF)derived porous carbon $[20,21]$, among others) are considered the best option. Notably, the most widely used is AC, which is characterized by a high specific surface area. With respect to the anode materials, the AC positive materials match the abundant micropores, and high-conductivity materials appear to be a rational choice. There are three main types of 
anode material: embedded materials (such as graphite and graphene [21-26]), conversion materials (e.g., $\mathrm{Fe}_{3} \mathrm{O}_{4}$ [27]), and alloys (including $\mathrm{Si} / \mathrm{Cu}$ and $\mathrm{SiO}_{2} / \mathrm{C}[28,29]$ ). Among these, metal oxides with a high specific capacitance have been widely used, whereas low conductivity and irreversible structural changes confine their rate index and stability [30].

Among transition metal oxides, $\mathrm{NiO}$ is considered to be ideal due to its excellent chemical and physical properties, such as its high theoretical capacitance and ease of processing. With respect to the quality of electrochemical properties [31,32], the synergistic relationship between graphene nanosheets and ultrafine $\mathrm{NiO}$ nanoparticles enables the development of materials with enhanced stability and rate performance. Regarding the electrochemical properties of NiO-rGO composite, this study found that after 200 cycles at a current density of $0.5 \mathrm{~A} \mathrm{~g}^{-1}$, the capacity retention rate was $95.6 \%$ and the coulomb efficiency was $\sim 100 \%$, indicating good cycle stability. The large specific surface area and adjustable pore structure of AC have made it the pre-eminent choice of positive material. Therefore, $\mathrm{AC}$ has been introduced alongside NiO-rGO for the construction of positive and negative materials for LICs. In this study, an LIC was rationally designed using a $\mathrm{NiO}-\mathrm{rGO}$ composite as the negative material and commercial $\mathrm{AC}$ as the positive material. Additionally, a NiO-rGO/ / AC LIC system with a mass ratio of 1:2 to 1:4 (negative/positive) was successfully fabricated. LICs with a mass ratio of 1:3 achieved a $122 \mathrm{~W} \mathrm{~h} \mathrm{~kg}^{-1}$ energy density, a $32.3 \mathrm{~kW} \mathrm{~kg}^{-1}$ power density, and a capacity retention of $72.1 \%$ after 10,000 cycles.

\section{Experiment}

\subsection{Synthesis of $G O$}

The graphite oxide utilized in this experiment was prepared by means of the traditional Hummer method. First, graphite powder (2 g), $\mathrm{NaNO}_{3}(2 \mathrm{~g})$, and concentrated $\mathrm{H}_{2} \mathrm{SO}_{4}$ $(92 \mathrm{~mL})$ were mixed and stirred for $4 \mathrm{~h}$. Next, $12 \mathrm{~g}$ of $\mathrm{KMnO}_{4}$ was added and stirred at $25^{\circ} \mathrm{C}$ for $2 \mathrm{~h}$. Then, $184 \mathrm{~mL}$ of deionized water was then slowly added to induce a full reaction. After that, $40 \mathrm{~mL}$ of $\mathrm{H}_{2} \mathrm{O}_{2}$ was added, and stirred for 15 min. Finally, it was washed, centrifuged, and dried, after which the preparation of the GO was complete.

\subsection{Synthesis of the NiO-rGO Composite}

The NiO-rGO composite was obtained by means of a simple hydrothermal reaction. First, $62 \mathrm{~g}$ of $\mathrm{NiSO}_{4}$ was dissolved in $50 \mathrm{~mL}$ deionized water, stirring for $15 \mathrm{~min}$ to fully dissolve it. Next, $4 \mathrm{~mL}$ of ammonia water $(25 \%)$ was slowly added to the $\mathrm{NiSO}_{4}$ and then dispersed by ultrasound for $30 \mathrm{~min}$. Through the above steps, solution A was obtained. Then, $20 \mathrm{mg}$ of GO was dissolved in $50 \mathrm{~mL}$ deionized water. By dispersing the mixture using ultrasound for $30 \mathrm{~min}$, solution B was obtained. Solution A was then slowly added to solution $B$ and stirred for 20 min to obtain solution C. Subsequently, $8 \mathrm{mg} \mathrm{mL}^{-1} \mathrm{NaBH}_{4}$ was carefully added to solution $\mathrm{C}$, which was then stirred for $1 \mathrm{~h}$ and heated in a water bath at $80{ }^{\circ} \mathrm{C}$ for $12 \mathrm{~h}$. The mixed solution was cooled to $25^{\circ} \mathrm{C}$, washed, and dried with deionized water. The final product was the NiO-rGO composite.

\subsection{Characterization}

The X-ray diffraction (XRD) properties of the crystal were measured using $\mathrm{Cu} \mathrm{K} \alpha$ $(\lambda=0.15406 \mathrm{~nm})$ radiation on a D8 focusing diffractometer (Bruker D8 DISCOVER, Bruker, Leipzig, Germany). The morphology of the materials was tested by means of a scanning electron microscope (SEM, Nova NanoSEM 450, FEI, Hillsborough, Oregon, OR, USA), energy-dispersive X-ray spectroscopy (EDX, SciAps, Boston, MA, USA), and transmission electron microscopy (TEM, JEOL JSM-2010, JEOL, Tokyo, Japan). The Raman spectra were determined using a LabRAM HR Raman spectrometer (Horiba, Kyoto, Japan). The X-ray photoelectron spectroscopy (XPS) spectra of the materials were measured using ESCALAB 250Xi (Thermo Fisher, Waltham, MA, USA). 


\subsection{Electrochemical Measurements}

The coating method was used for the preparation of the electrode. The active materials, Super C45 and polyvinylidene fluoride (PVDF), were blended in an $\mathrm{N}$-methyl-2pyrrolidone (NMP) solution at a ratio of 8:1:1. The weight ratio of NMP was approximately $75-80 \%$. After that, the mixed solution of negative and positive materials was evenly coated onto a collector of copper foil (negative) or aluminum foil (positive) by means of a coater. The coated foil was then cut into sheets of $10 \mathrm{~mm}$ (negative electrode) or $13 \mathrm{~mm}$ (positive electrode) after drying. A working electrode was obtained by vacuum drying the resulting product overnight at $120^{\circ} \mathrm{C}$. A button cell was assembled using lithium foil as the counter electrode in a 3-electrode system and polyethylene as a separator. The electrolyte was $1 \mathrm{M} \mathrm{LiPF}_{6}$, which was blended in a mixed solvent of dimethyl carbonate (DMC), diethyl carbonate (DEC), and ethylene carbonate at a volume ratio of 1:1:1. The mass load for a single electrode was $1 \mathrm{mg} \mathrm{cm}^{-2}$.

The LICs were assembled utilizing a pre-embedded lithium negative and pristine positive 2-electrode cell. The ratios of the mass of the active materials of the negative (NiOrGO) and positive (AC) electrodes were 1:2, 1:3, and 1:4. Their electrochemical performance, including pre-lithiation, galvanostatic charge-discharge progress, and rate and cycle life, were measured using a Neware battery-testing device. Cyclic voltammetry (CV) curves were determined using a BioLogic VMP3 electrochemical station (Biologic, Sohn, France). The calculation process of energy density, specific capacitance, and power density (based on the total mass of the two electrode materials) was as follows:

$$
\begin{gathered}
C=\frac{\int I d V}{2 m \Delta V v} \\
E=\int_{t 1}^{t 2} I \mathrm{~V} d t=\frac{1}{2} C\left(\mathrm{~V}_{\max }+\mathrm{V}_{\min }\right)\left(\mathrm{V}_{\max }-\mathrm{V}_{\min }\right) \\
P=\frac{E}{t}
\end{gathered}
$$

where $I$ is the current, $v$ is the scanning speed, $\mathrm{V}$ is the voltage, $v$ is the scanning speed used in the CV test, $t$ is the discharge time, $m$ is the total mass of electrode materials, and $V_{\max }$ and $V_{\min }$ are the voltage at the start and the end of discharge, respectively.

\section{Results and Discussion}

\subsection{Material Performance Characterization}

In the XRD pattern (Figure 1), the diffraction peak at $24.5^{\circ}$ was the characteristic peak of rGO. The main diffraction peaks of the NiO-rGO composite appeared at $37.2^{\circ}, 43.3^{\circ}$, $62.8^{\circ}, 75.4^{\circ}$, and $79.3^{\circ}$, corresponding to crystal plane diffractions of (111), (200), (220), (311), and (222) a.u., respectively, and aligning with $\mathrm{NiO}^{\prime}$ s standard card, with no other peaks emerging. This further demonstrated that the NiO-rGO composite, having been prepared following the aforementioned method, had a high crystal purity, with no other substances appearing.

The layered rGO was well preserved and the thickness of the layered composite was less than $10 \mathrm{~nm}$ (Figure 2a,b), which indicated that the number of rGO layers was small and that the rGO sheet did not agglomerate because $\mathrm{NiO}$ nanoparticles were uniformly anchored in it. Furthermore, there was no serious $\mathrm{NiO}$ aggregation and all $\mathrm{NiO}$ nanoparticles were well anchored to the rGO, indicating that the morphology of the composite was good. Figure $2 \mathrm{c}$ displays uniform $\mathrm{NiO}$ nanoparticles in the size of ten to tens of nanometers. Figure $2 \mathrm{~d}$ shows that the $\mathrm{NiO}$ crystal size was about $5.5 \pm 1.5 \mathrm{~nm}$ and the crystal plane spacing of $\mathrm{NiO}$ nanoparticles was about $0.24 \mathrm{~nm}$, corresponding to the (111) plane of the cubic $\mathrm{NiO}$ crystal. An EDX analysis (Figure 2e) revealed that the distribution of $\mathrm{Ni}$ and $\mathrm{O}$ was relatively uniform, and that there was no obvious aggregation. Moreover, the $\mathrm{C}, \mathrm{Ni}$, and $\mathrm{O}$ content was $78 \%, 12 \%$, and $10 \%$, respectively. Therefore, the NiO-rGO composite could be synthesized without additional material doping. 


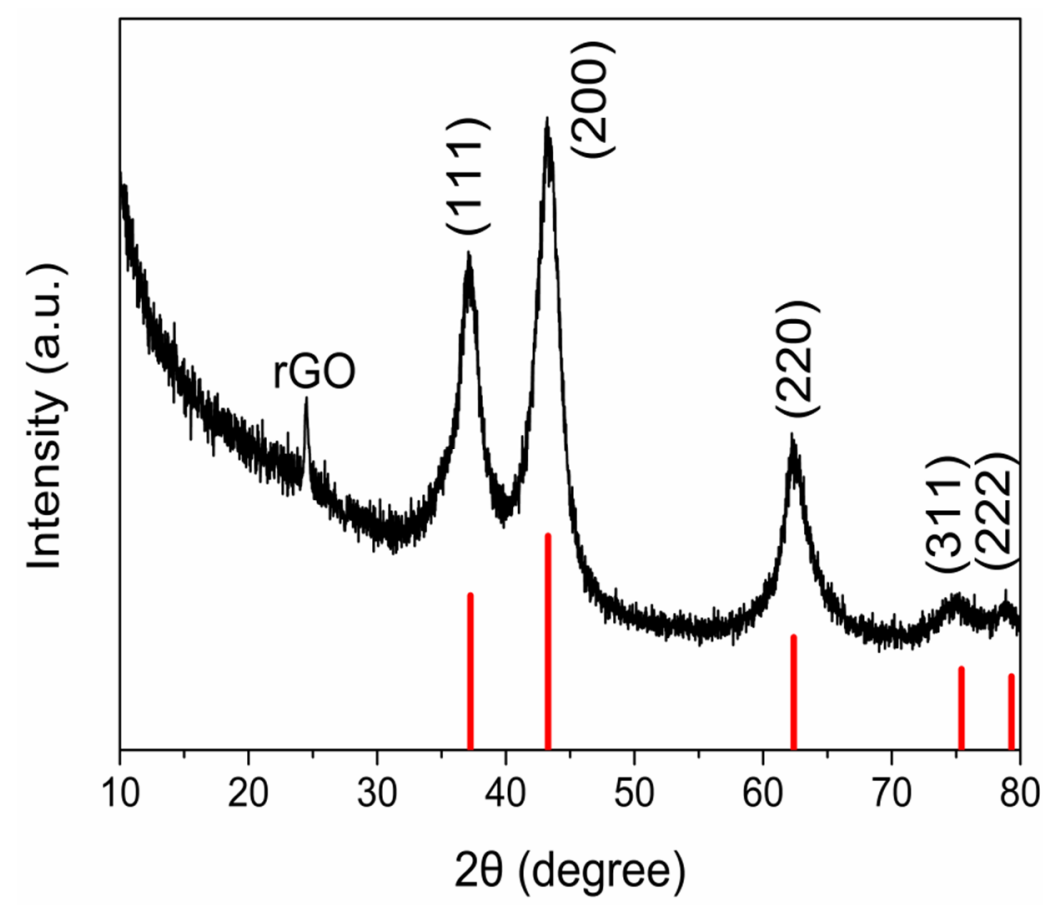

Figure 1. XRD pattern of the NiO-rGO composite.
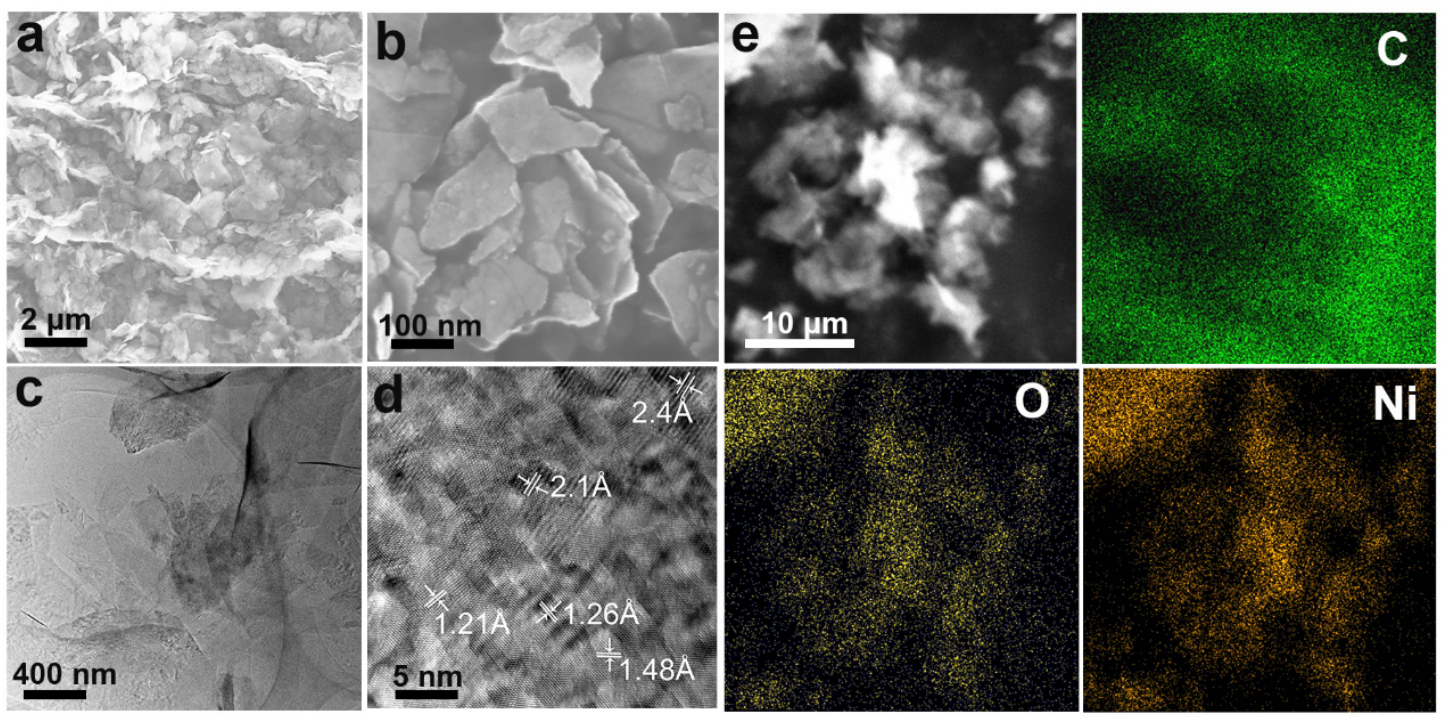

Figure 2. (a,b) SEM of the NiO-rGO composite. (c,d) TEM of the NiO-rGO composite. (e) EDX mapping (C, O, Ni) of the NiO-rGO composite.

An XPS scan was used to evaluate the surface electronic states of the elements. Figure $3 a$ displays the $\mathrm{Ni} 2 \mathrm{p}, \mathrm{C} 1 \mathrm{~s}$, and $\mathrm{O} 1$ s peaks that confirm the coexistence of $\mathrm{NiO}$ and rGO in the composite. In the $\mathrm{Ni} 2 \mathrm{p}$ spectra (Figure $3 \mathrm{~b}$ ), the peaks at $855.8 \mathrm{eV}$ and $873.6 \mathrm{eV}$ belong to the $\mathrm{Ni} 2 \mathrm{p}_{2 / 3}$ and $\mathrm{Ni} 2 \mathrm{p}_{1 / 2}$, respectively. The $\mathrm{C} 1$ s spectra (Figure $3 \mathrm{c}$ ) were fitted to four peaks of $284.7 \mathrm{eV}, 285.4 \mathrm{eV}, 287.0 \mathrm{eV}$, and $289.9 \mathrm{eV}$, corresponding to $\mathrm{C}=\mathrm{C}, \mathrm{C}-\mathrm{OH}, \mathrm{C}-\mathrm{O}-\mathrm{C}$, and $\mathrm{O}-\mathrm{C}=\mathrm{O}$, respectively. The $\mathrm{O} 1$ s spectrum (Figure $3 \mathrm{~d}$ ) showed three main peaks, with the one at $531.8 \mathrm{eV}$ corresponding to $\mathrm{rGO}$, and those at $531.2 \mathrm{eV}$ and $529.3 \mathrm{eV}$ both corresponding to $\mathrm{NiO}$. Based on the above discussion, the bond between nickel and an oxygen-containing functional group may be the result of the combination of $\mathrm{NiO}$ nanocrystals and rGO nanoplates [33]. 

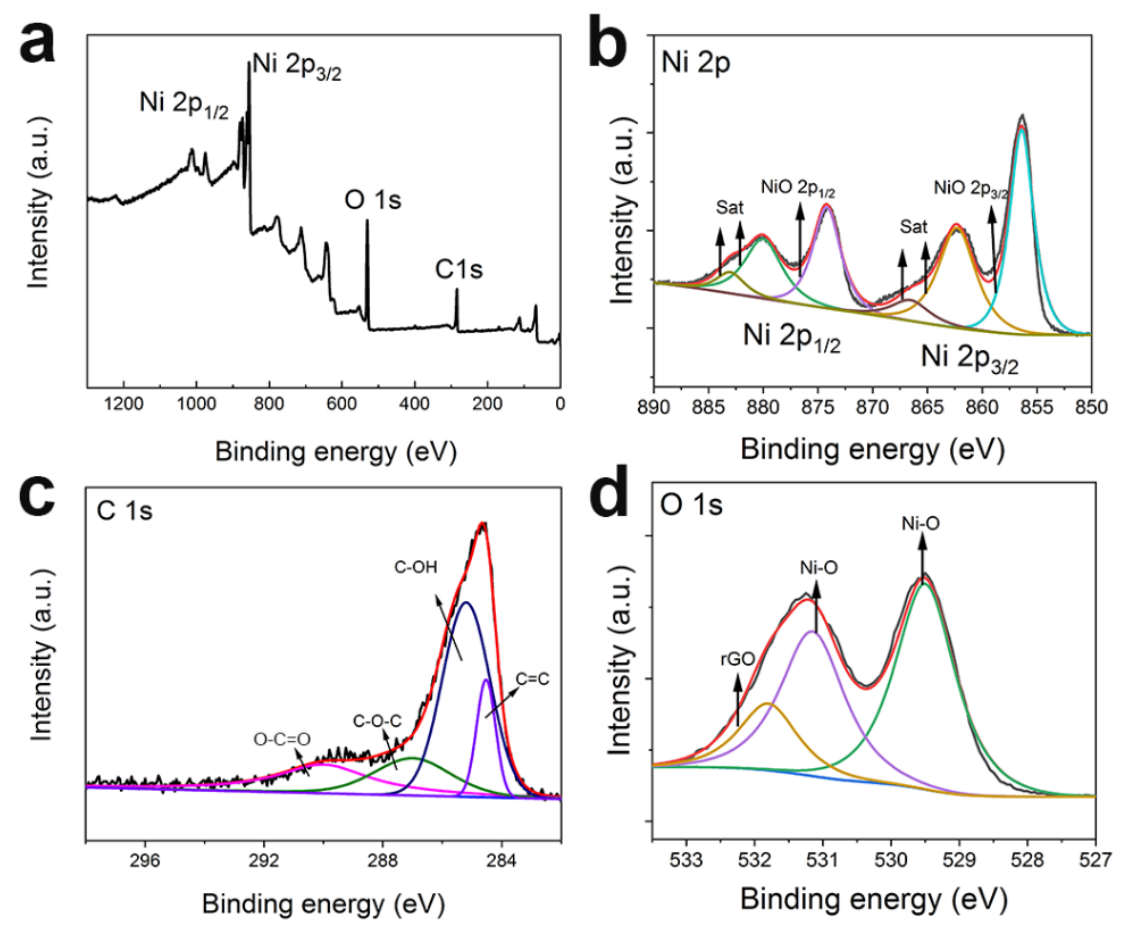

Figure 3. (a) XPS and high-resolution spectra of (b-d) Ni 2p, C 1s, and O 1s peaks in the NiO-rGO composite.

Figure 4a presents the $\mathrm{N}_{2}$ adsorption/desorption isotherm and pore size distribution of NiO-rGO. The specific surface area of the NiO-rGO composite was $89.7 \mathrm{~m}^{2} \mathrm{~g}^{-1}$, lower than that of $\mathrm{rGO}$ (about $204.3 \mathrm{~m}^{2} \mathrm{~g}^{-1}$ ), due to the much heavier weight of the $\mathrm{NiO}$ compared to the rGO. The mesopores (concentrated at $4 \mathrm{~nm}$ ) can be observed in the graph, and can be attributed to the interaction between the $\mathrm{NiO}$ and $\mathrm{rGO}$.
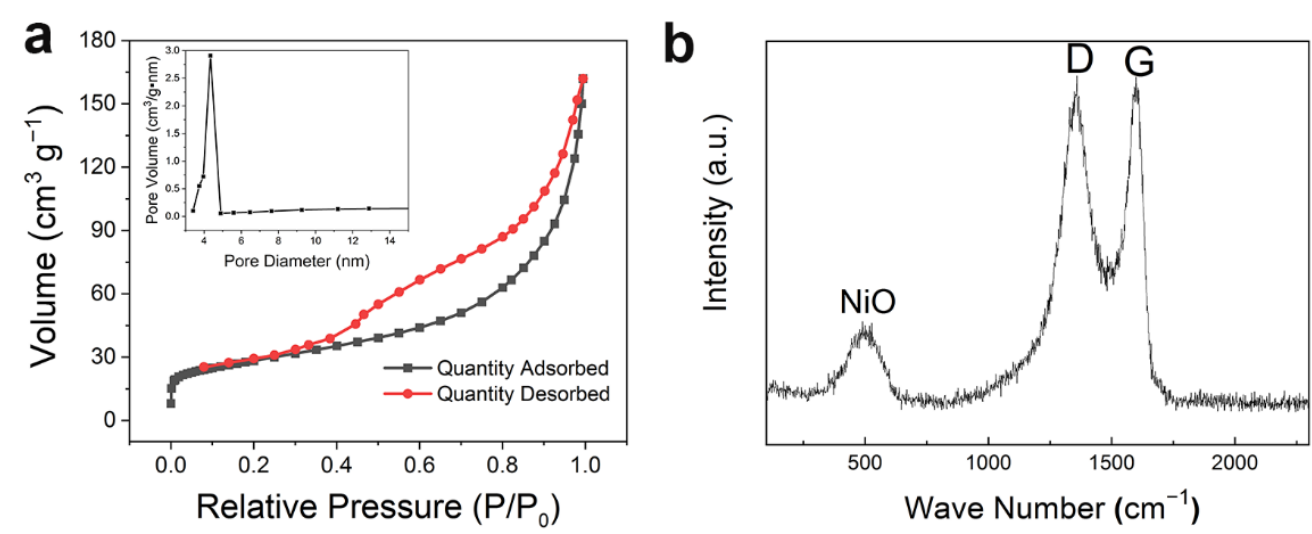

Figure 4. (a) $\mathrm{N}_{2}$ adsorption-desorption isotherm and pore size distribution (BJH) model of the NiO-rGO composite; (b) Raman spectra of the NiO-rGO composite.

The Raman spectra of the composite (Figure $4 b$ ) indicate that there were two peaks of $C$ at $1357 \mathrm{~cm}^{-1}$ (D peak) and $1598 \mathrm{~cm}^{-1}$ (G peak), and the characteristic peak of $\mathrm{NiO}$ appeared at $500 \mathrm{~cm}^{-1}$. Researchers often use the ratio of the $\mathrm{D}$ to $\mathrm{G}$ peak $\left(I_{\mathrm{D}} / I_{\mathrm{G}}\right)$ as the index for detecting the defect density. The $I_{\mathrm{D}} / I_{\mathrm{G}}$ of the NiO-rGO composite was 0.96 , which indicated that abundant defects were introduced during its synthesis. The existence of defects can improve the ion/electron transport speed, improve the conductivity, and further improve the electrochemical performance. 


\subsection{Performance of Half-Cells}

To evaluate the properties of the NiO-rGO composite, constant current charge and discharge were tested under $0.05-3 \mathrm{~V}$ (vs. $\mathrm{Li} / \mathrm{Li}^{+}$).

Figure $5 \mathrm{a}$ depicts the $\mathrm{CV}$ curves $\left(0.05-3 \mathrm{~V}, 0.1 \mathrm{mV} \mathrm{s}^{-1}\right)$. During the positive process, an obvious peak appeared at $\sim 0.5 \mathrm{~V}$ due to the formation of a solid electrochemical interface (SEI) film. The second and third curves essentially coincided, indicating the high stability of the material. Figure $5 \mathrm{~b}$ shows the charge/discharge curves of the NiO-rGO composite during the first four cycles at $0.1 \mathrm{~A} \mathrm{~g}^{-1}$ and $0.05-3.0 \mathrm{~V}\left(\mathrm{vs}\right.$. Li/ $\left.\mathrm{Li}^{+}\right)$. The initial discharge capacity and charging capacity of the NiO-rGO composite were $2700 \mathrm{~mA} \mathrm{~h} \mathrm{~g}^{-1}$ and $1644 \mathrm{~mA} \mathrm{~h} \mathrm{~g}^{-1}$, respectively: higher than the theoretical capacity of $\mathrm{NiO}\left(718 \mathrm{~mA} \mathrm{~h} \mathrm{~g}^{-1}\right)$. The formation of an SEI film during the first charge and discharge consumes a lot of lithium ions, which leads to a large difference in specific capacity between charge and discharge. In later charge and discharge processes, it gradually stabilizes. Figure $5 c$ displays the rate performance at $0.5-4 \mathrm{~A} \mathrm{~g}^{-1}$. When at $0.5 \mathrm{~A} \mathrm{~g}^{-1}$, the charge capacity was $945.8 \mathrm{~mA} \mathrm{~h} \mathrm{~g}^{-1}$. This slower reaction rate is more conducive to the diffusion of lithium ions into NiO-rGO, leading to a high specific capacity. At $4 \mathrm{~A} \mathrm{~g}^{-1}$, the charge capacity was $275.6 \mathrm{~mA} \mathrm{~h} \mathrm{~g}^{-1}$, which related to the crystallinity and size of the nanocrystals. When charging and discharging at higher currents, the speed of lithium-ion intercalation and exfoliation in the electrode material is faster, and crystal lattice distortion is more likely to occur, leading to crystal shedding and electrochemical performance degradation. The charge-discharge reaction is gradually balanced when the current reverts to $0.5 \mathrm{~A} \mathrm{~g}^{-1}$. A capacity of $897.3 \mathrm{~mA} \mathrm{~h} \mathrm{~g}^{-1}$ can be obtained, which is better than, or comparable to the reported data (high specific capacity of $520 \mathrm{~mA} \mathrm{~h} \mathrm{~g}^{-1}$ at $2400 \mathrm{~mA} \mathrm{~g}^{-1}$ ) [34]. This shows that the NiO-rGO composite has good reversible cycle performance and high reversible capacity at high current density. Figure $5 \mathrm{~d}$ shows that, after 200 cycles at a current density of $0.5 \mathrm{~A} \mathrm{~g}^{-1}$, the capacity retention rate was $95.6 \%$ and the coulomb efficiency was nearly $100 \%$, indicating good cycle stability. Electrochemical impedance spectroscopy (EIS) was performed in the frequency range of $100 \mathrm{kHz}$ to $0.01 \mathrm{~Hz}$ with an amplitude of $5 \mathrm{mV}$. The EIS spectrum appears as a semicircle at high frequency and has a linear part at low frequency. In the high frequency range, the intercept on the real axis is the volume solution resistance (Re), and the diameter of the semicircle on the real axis is approximately equal to the charge transfer resistance (Rct). The oblique line in the low-frequency region indicates the ion diffusion process in the electrode. As shown in Figure S1, the Re of NiO-rGO is significantly lower than that of $\mathrm{NiO}$, its corresponding straight line is steeper than the one for $\mathrm{NiO}$, and the ion diffusion on the surface is faster. The abovementioned excellent properties were mainly due to the presence of $\mathrm{rGO}$, which not only enhances ion and electron transmission, but also reduces the structural collapse of metal oxides during the intercalation and desorption of lithium ions. 

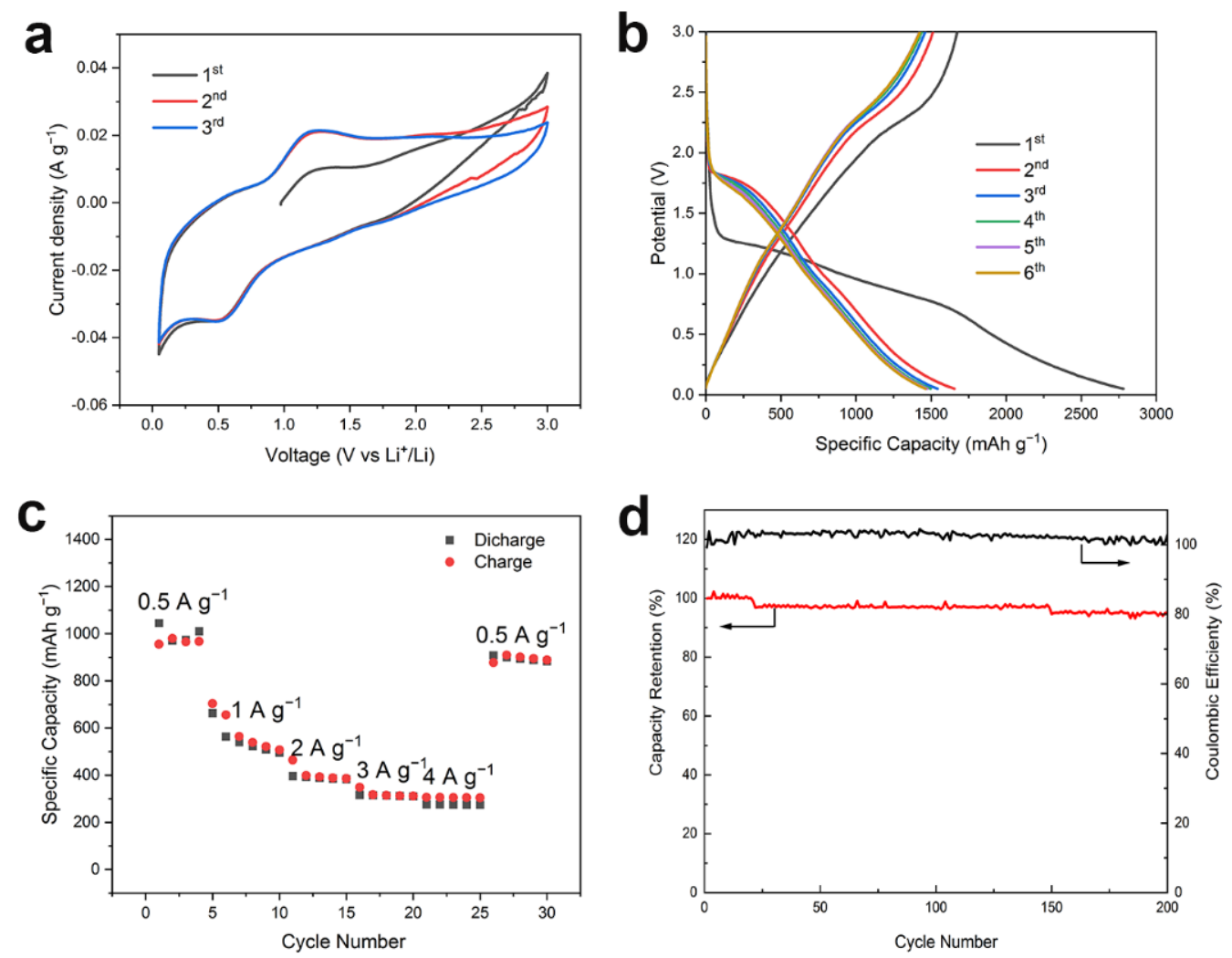

Figure 5. Electrochemical performance of the NiO-rGO. (a) $\mathrm{CV}$ curves at $0.1 \mathrm{mV} \mathrm{s}^{-1}$. (b) First four charge-discharge curves at $0.1 \mathrm{~A} \mathrm{~g}^{-1}$. (c) Rate performance. (d) Cyclic behavior at $0.5 \mathrm{~A} \mathrm{~g}^{-1}$.

\subsection{Electrochemical Performance of LICs}

In this study, LICs were prepared using AC as the positive electrode and pre-lithiated $\mathrm{NiO}-\mathrm{rGO}$ as the negative electrode, with a mass ratio of 1:2 to 1:4 (negative-to-positive). Based on this, the electrochemical performance of the LICs was optimized.

The charge storage mechanism is shown in Figure 6a. During charging, $\mathrm{PF}_{6}{ }^{-}$ions were transported to the AC positive electrode, whereas $\mathrm{Li}^{+}$ions were inserted on the surface of the NiO-rGO negative electrode. The discharging procedure was in contrast to the charging process. Figure $6 \mathrm{~b}$ shows the $\mathrm{CV}$ curve of the LICs with a mass ratio of 1:3. At the scanning rates of 10,50 , and $100 \mathrm{mV} / \mathrm{s}^{-1}$, the curves were nearly rectangular, indicating that the system had attained a good capacitance performance and rate capability. The energy storage mechanism of $\mathrm{NiO}$ is such that with the increase in scanning speed, the rectangular shape of $\mathrm{CV}$ curve is difficult to maintain. Figure $6 \mathrm{c}$ displays the constant current charge-discharge curve under different current densities (the mass ratio was 1:3, 1.0-4.0 V). At $0.5 \mathrm{~A} \mathrm{~g}^{-1}$, the middle section of the charge curve was curved, which was due to the insertion and extraction of the lithium ions; at $1.5 \mathrm{~A} \mathrm{~g}^{-1}$, the curve exhibited triangular symmetry, which conforms to the capacitance characteristics. Furthermore, no significant IR drop occurred in these curves, indicating that the internal resistance of the system was very low. Due to the difference in the specific capacitance of positive and negative materials, in order to optimize the voltage window and obtain the best performance, it is necessary to adjust the active material weight ratio of the positive and negative electrodes. Figure $6 \mathrm{~d}$ demonstrates the rate capability of the NiO-rGO/ / AC system. It can be noted that LICs with negativeto-positive mass ratios of 1:3 feature optimized performance. Moreover, the capacitance of the NiO-rGO/ / AC system was $43.75 \mathrm{Fg}^{-1}$ (at $0.2 \mathrm{~A} \mathrm{~g}^{-1}$ ) (based on the proportion of active substances). The NiO-rGO/ / AC hybrid system was compared to other capacitors (Table S1). Compared to other hybrid systems, NiO-rGO// AC has major advantages, and it can achieve greater energy combination and maintain satisfactory energy density at a high power output (Figure 6e). To assess its practical applications, a cycle-life test 
was performed. Figure $6 \mathrm{f}$ indicates that the capacity retention rate of $\mathrm{NiO}-\mathrm{rGO} / / \mathrm{AC}$ was $76.1 \%$ after 10,000 cycles, and the coulomb efficiency remained between $99 \%$ and $100 \%$, demonstrating good cycle stability. The decrease in the cycle capacity was primarily due to the negative electrode, the volume of which changed greatly during the charging and discharging processes. Lattice distortion leads to a decrease in conductivity and shedding of active substances.
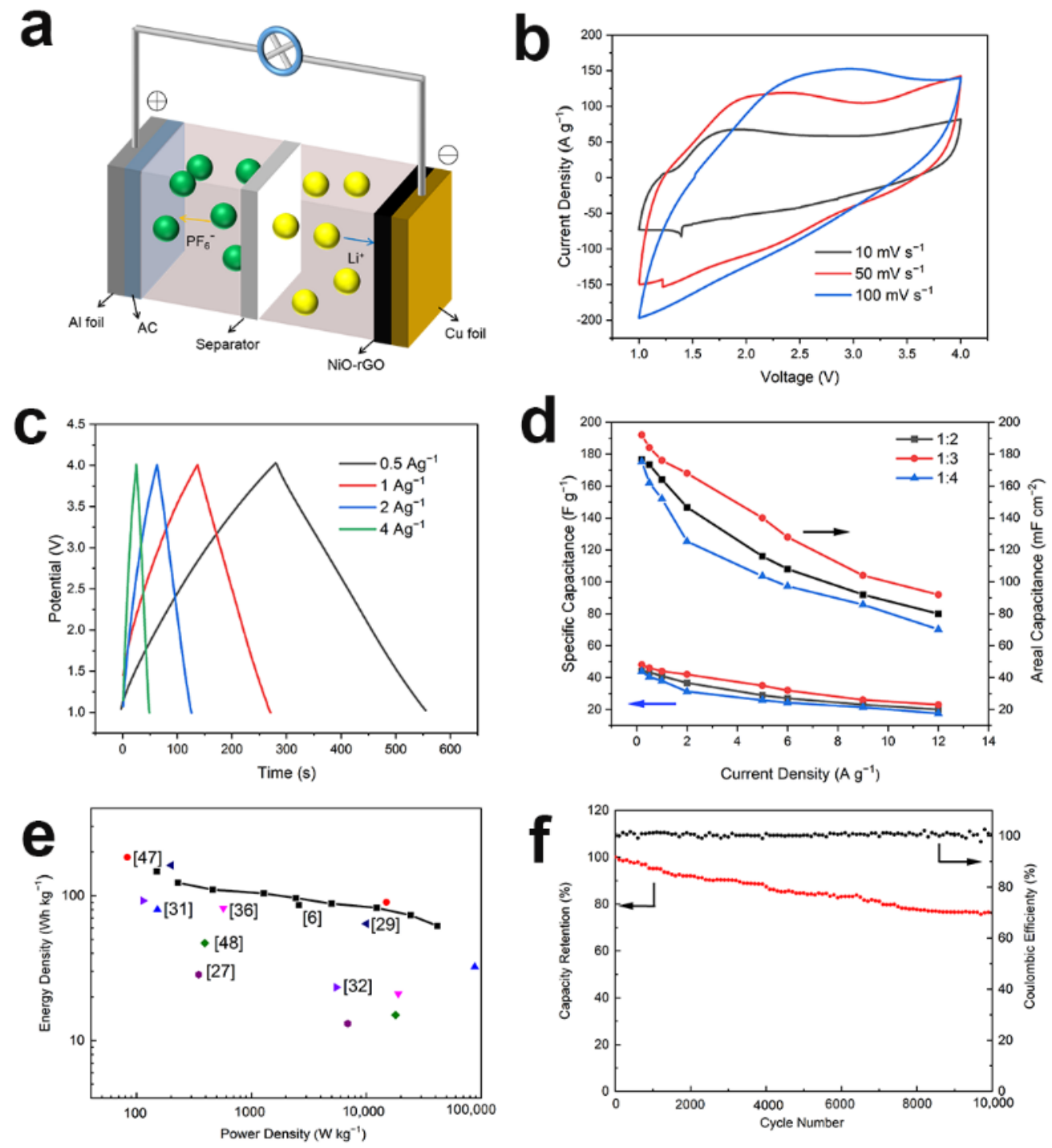

Figure 6. (a) Schematic diagram of working mechanism for the NiO-rGO// AC system. (b) CV curves. (c) Galvanostatic charge-discharge curves with a mass ratio of 1:3. (d) Specific capacitance (left axis) and area capacitance (right axis) of NiO-rGO// AC. (e) Ragone plots (mass ratio: 1:3), in comparison with recent literature. (f) Cycle life and coulombic efficiency curves of LICs with a mass ratio of 1:4, the inset of which was a demonstration of NiO-rGO/ / AC.

The unique performance of the NiO-rGO// AC system can be summarized as follows: (i) the presence of rGO can enhance conductivity, facilitate the transmission of ions and electrons, and reduce the lattice distortion of metal oxides caused by the insertion and extraction of lithium ions; (ii) a sufficient interface is provided by AC to accumulate opposing charges; and (iii) the proper ratio of positive to negative contributes to obtaining the best output performance. 


\subsection{The Prospect of Industrialization}

Laboratory research into the development of new materials has been unable to keep pace with the needs of industry. Bottlenecks are caused by a variety of problems: the high cost of materials hampers large-scale production, the preparation and purification of certain materials produces toxic substances, there are difficulties with recycling used materials, etc. The research presented here highlights a number of opportunities to circumvent these barriers. Firstly, regarding cost, the prices of rGO and NiO are moderate. Secondly, regarding the preparation process, this study used the well-established Hummer method to prepare the $\mathrm{rGO}$, while the NiO-rGO composite was prepared using the hydrothermal method and low-temperature heat treatment; these preparation processes have low environmental impacts and require little in the way of specialized equipment. This has the potential to lower production costs when scaled up to industrial production. Finally, the electrochemical performance of the system is excellent compared to established designs. Therefore, the LIC design presented in this paper offers many advantages when it comes to future commercial production.

\section{Conclusions}

In summary, an LIC was successfully designed using a NiO-rGO composite as the negative material and commercial $\mathrm{AC}$ as the positive material, producing a battery with excellent electrochemical performance. It was shown that the integration of $\mathrm{NiO}$ with carbon-based materials can improve the electrochemical performance in LIC applications. Furthermore, the presence of graphene enhances the transport of ions and electrons and effectively suppresses the volume expansion of the metal oxide when lithium ions are inserted into or extracted from the electrode material. In addition, the NiO-rGO composite featured a larger specific surface area and a nanoscale ion diffusion pathway, which was conducive to the insertion and extraction of lithium ions. The NiO-rGO composite had good reversible cycling performance at both low and high current densities, and also had a high reversible capacity at high current densities. In addition, the NiO-rGO/ / AC LIC system with a mass ratio of 1:2-1:4 (negative/positive) was synergistically fabricated. The LIC with a mass ratio of 1:3 achieved excellent electrochemical performance, with an energy density of $98.15 \mathrm{~W} \mathrm{~h} \mathrm{~kg}^{-1}$, a power density of $10.94 \mathrm{~kW} \mathrm{~kg}^{-1}$, a reasonable level of cycle stability, and a capacity retention of $72.1 \%$ after 10,000 cycles. The rationally designed NiO-rGO/ / AC system presents a number of superb opportunities in the development of high-performance LICs.

Supplementary Materials: The following are available online at https://www.mdpi.com/article/ 10.3390/ma14133586/s1, Figure S1: Nyquist plots of NiO-rGO electrodes in the frequency range of $100 \mathrm{kHz}-0.01 \mathrm{~Hz}$ and the inset is the enlarged EIS, Table S1: Electrochemical performances of recently published Li-ion hybrid capacitors in organic system.

Author Contributions: Conceptualization and methodology, Q.A., X.Z. and S.S.; Validation, Q.A. and Y.B.; Writing-Original Draft Preparation and Visualization, Q.A. All authors have read and agreed to the published version of the manuscript.

Funding: This work was financially supported by the National Key Research and Development Program of China (No. 2017YFF0108101).

Conflicts of Interest: The authors declare no conflict of interest.

\section{References}

1. Liu, C.; Li, F.; Ma, L.-P.; Cheng, H.-M. Advanced Materials for Energy Storage. Adv. Mater. 2010, 22, E28-E62. [CrossRef]

2. Huang, X.; Yin, Z.; Wu, S.; Qi, X.; He, Q.; Zhang, Q.; Yan, Q.; Boey, F.; Zhang, H. Graphene-Based Materials: Synthesis, Characterization, Properties, and Applications. Small 2011, 7, 1876-1902. [CrossRef]

3. Yuan, C.; Wu, H.; Xie, Y.; Lou, X.W. Mixed Transition-Metal Oxides: Design, Synthesis, and Energy-Related Applications. Angew. Chem. Int. Ed. 2014, 53, 1488-1504. [CrossRef] [PubMed]

4. Lai, X.; Halpert, J.E.; Wang, D. Recent advances in micro-/nano-structured hollow spheres for energy applications: From simple to complex systems. Energy Environ. Sci. 2012, 5, 5604-5618. [CrossRef] 
5. Liu, C.; He, Z.; Niu, J.; Cheng, Q.; Zhao, Z.; Li, H.; Shi, J.; Wang, H. Two-dimensional SnO 2 anchored biomass-derived carbon nanosheet anode for high-performance Li-ion capacitors. RSC Adv. 2021, 11, 10018-10026. [CrossRef]

6. Luo, X.; Yang, J.; Yan, D.; Wang, W.; Wu, X.; Zhu, Z. $\mathrm{MnO}_{2}$-decorated 3D porous carbon skeleton derived from mollusc shell for high-performance supercapacitor. J. Alloys Compd. 2017, 723, 505-511. [CrossRef]

7. Pal, B.; Vijayan, B.L.; Krishnan, S.G.; Harilal, M.; Basirun, W.J.; Lowe, A.; Yusoff, M.M.; Jose, R. Hydrothermal syntheses of tungsten doped $\mathrm{TiO}_{2}$ and $\mathrm{TiO}_{2} / \mathrm{WO}_{3}$ composite using metal oxide precursors for charge storage applications. J. Alloys Compd. 2018, 740, 703-710. [CrossRef]

8. Vijayan, B.L.; Misnon, I.I.; Anilkumar, G.M.; Yang, C.-C.; Jose, R. Void-size-matched hierarchical 3D titania flowers in porous carbon as an electrode for high-density supercapacitive charge storage. J. Alloys Compd. 2021, 858, 157649. [CrossRef]

9. Lyu, L.; Kang, J.; Seong, K.-D.; Kim, C.W.; Lim, J.; Piao, Y. ZnNiCo hydroxide/graphene-carbon nanotube hydrogel on surfacemodified Ni foam as a battery-type electrode for hybrid supercapacitors. J. Alloys Compd. 2021, 872, 159610. [CrossRef]

10. Yang, B.; Chen, J.; Liu, B.; Ding, Y.; Tang, Y.; Yan, X. One dimensional graphene nanoscroll-wrapped MnO nanoparticles for high-performance lithium ion hybrid capacitors. J. Mater. Chem. A 2021, 9, 6352-6360. [CrossRef]

11. Kavinkumar, T.; Seenivasan, S.; Lee, H.H.; Jung, H.; Han, J.W.; Kim, D.-H. Interface-modulated uniform outer nanolayer: A category of electrodes of nanolayer-encapsulated core-shell configuration for supercapacitors. Nano Energy 2021, 81, 105667. [CrossRef]

12. Li, H.; Hu, Z.; Xia, Q.; Zhang, H.; Li, Z.; Wang, H.; Li, X.; Zuo, F.; Zhang, F.; Wang, X.; et al. Operando Magnetometry Probing the Charge Storage Mechanism of CoO Lithium-Ion Batteries. Adv. Mater. 2021, 33, 2006629. [CrossRef] [PubMed]

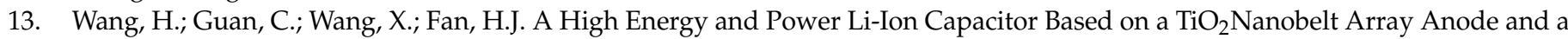
Graphene Hydrogel Cathode. Small 2015, 11, 1470-1477. [CrossRef] [PubMed]

14. Yan, W.; Su, J.; Yang, Z.; Lv, S.; Jin, Z.; Zuo, J. High-Performance Lithium-Ion Capacitors Based on Porosity-Regulated Zirconium Metal-Organic Frameworks. Small 2021, 17, e2005209. [CrossRef] [PubMed]

15. Dutta, S.; Bhaumik, A.; Wu, K.C.-W. Hierarchically porous carbon derived from polymers and biomass: Effect of interconnected pores on energy applications. Energy Environ. Sci. 2014, 7, 3574-3592. [CrossRef]

16. Wang, X.; Lu, X.; Liu, B.; Chen, D.; Tong, Y.; Shen, G. Flexible Energy-Storage Devices: Design Consideration and Recent Progress. Adv. Mater. 2014, 26, 4763-4782. [CrossRef]

17. Tang, Z.; Tang, C.-H.; Gong, H. A High Energy Density Asymmetric Supercapacitor from Nano-architectured Ni(OH)2/Carbon Nanotube Electrodes. Adv. Funct. Mater. 2012, 22, 1272-1278. [CrossRef]

18. Zuo, W.; Li, R.; Zhou, C.; Li, Y.; Xia, J.; Liu, J. Battery-Supercapacitor Hybrid Devices: Recent Progress and Future Prospects. Adv. Sci. 2017, 4, 1600539. [CrossRef]

19. Yu, L.; Hu, H.; Bin Wu, H.; Lou, X.W.D. Complex Hollow Nanostructures: Synthesis and Energy-Related Applications. Adv. Mater. 2017, 29, 1604563. [CrossRef]

20. Shen, L.; Yu, L.; Yu, X.-Y.; Zhang, X.; Lou, X.W. Self-Templated Formation of Uniform $\mathrm{NiCo}_{2} \mathrm{O}_{4}$ Hollow Spheres with Complex Interior Structures for Lithium-Ion Batteries and Supercapacitors. Angew. Chem. Int. Ed. 2015, 54, 1868-1872. [CrossRef]

21. Guan, B.Y.; Yuan, G.B.; Bin Wu, H.; Lou, X.W. Complex Nanostructures from Materials based on Metal-Organic Frameworks for Electrochemical Energy Storage and Conversion. Adv. Mater. 2017, 29, 1703614. [CrossRef]

22. Pikul, J.H.; Zhang, H.; Cho, J.; Braun, P.V.; King, W. High-power lithium ion microbatteries from interdigitated three-dimensional bicontinuous nanoporous electrodes. Nat. Commun. 2013, 4, 1732. [CrossRef]

23. Luo, J.; Zhang, W.; Yuan, H.; Jin, C.; Zhang, L.; Huang, H.; Liang, C.; Xia, Y.; Zhang, J.; Gan, Y.; et al. Pillared Structure Design of MXene with Ultralarge Interlayer Spacing for High-Performance Lithium-Ion Capacitors. ACS Nano 2017, 11, 2459-2469. [CrossRef]

24. Han, X.; Han, P.; Yao, J.; Zhang, S.; Cao, X.; Xiong, J.; Zhang, J.; Cui, G. Nitrogen-doped carbonized polyimide microsphere as a novel anode material for high performance lithium ion capacitors. Electrochimica Acta 2016, 196, 603-610. [CrossRef]

25. Zhao, Y.; Hu, L.; Zhao, S.; Wu, L. Preparation of $\mathrm{MnCo}_{2} \mathrm{O}_{4} @ \mathrm{Ni}(\mathrm{OH})_{2}$ Core-Shell Flowers for Asymmetric Supercapacitor Materials with Ultrahigh Specific Capacitance. Adv. Funct. Mater. 2016, 26, 4085-4093. [CrossRef]

26. Reddy, A.L.M.; Gowda, S.R.; Shaijumon, M.; Ajayan, P.M. Hybrid Nanostructures for Energy Storage Applications. Adv. Mater. 2012, 24, 5045-5064. [CrossRef] [PubMed]

27. Qi, H.; Cao, L.; Li, J.; Huang, J.; Ma, M.; Cheng, Y.; Wang, C.; Dang, H. Rice crust-like Fe3O4@C/rGO with improved extrinsic pseudocapacitance for high-rate and long-life Li-ion anodes. J. Alloys Compd. 2019, 804, 57-64. [CrossRef]

28. Wang, R.; Lang, J.; Zhang, P.; Lin, Z.; Yan, X. Fast and Large Lithium Storage in 3D Porous VN Nanowires-Graphene Composite as a Superior Anode Toward High-Performance Hybrid Supercapacitors. Adv. Funct. Mater. 2015, 25, 2270-2278. [CrossRef]

29. Yu, X.; Zhan, C.; Lv, R.; Bai, Y.; Lin, Y.; Huang, Z.-H.; Shen, W.; Qiu, X.; Kang, F. Ultrahigh-rate and high-density lithium-ion capacitors through hybriding nitrogen-enriched hierarchical porous carbon cathode with prelithiated microcrystalline graphite anode. Nano Energy 2015, 15, 43-53. [CrossRef]

30. Shin, W.H.; Jeong, H.M.; Kim, B.G.; Kang, J.K.; Choi, J.W. Nitrogen-Doped Multiwall Carbon Nanotubes for Lithium Storage with Extremely High Capacity. Nano Lett. 2012, 12, 2283-2288. [CrossRef]

31. Peng, S.; Li, L.; Tan, H.; Cai, R.; Shi, W.; Li, C.; Mhaisalkar, S.G.; Srinivasan, M.; Ramakrishna, S.; Yan, Q. MS2(M = Co and Ni) Hollow Spheres with Tunable Interiors for High-Performance Supercapacitors and Photovoltaics. Adv. Funct. Mater. 2014, mboxemph24, 2155-2162. [CrossRef] 
32. Geng, P.; Zheng, S.; Tang, H.; Zhu, R.; Zhang, L.; Cao, S.; Xue, H.; Pang, H. Transition Metal Sulfides Based on Graphene for Electrochemical Energy Storage. Adv. Energy Mater. 2018, 8, 8. [CrossRef]

33. Zhao, X.; Zhang, X.; Li, C.; Sun, X.; Liu, J.; Wang, K.; Ma, Y. High-Performance Lithium-Ion Capacitors Based on CoO-Graphene Composite Anode and Holey Carbon Nanolayer Cathode. ACS Sustain. Chem. Eng. 2019, 7, 11275-11283. [CrossRef]

34. Bindumadhavan, K.; Chang, P.-Y.; Yeh, M.-H.; Doong, R.-A. Ultra-small CoO nanocrystals anchored on reduced graphene oxide for enhanced lithium storage in lithium ion batteries. MRS Commun. 2017, 7, 236-244. [CrossRef] 